\section{Tiempo destinado a estar sentado y niveles de adiposidad ¿Cuál es su efecto sobre el desarrollo de diabetes mellitus tipo 2 ?}

\author{
FANNY PETERMANN ${ }^{1, \mathrm{a}, *}$, ALEX GARRIDO-MÉNDEZ2,b,*, \\ XIMENA DÍAZ-MARTÍNEZ ${ }^{3, \mathrm{c}}$, ANA MARÍA LEIVA ${ }^{4, \mathrm{~d}}$, \\ MARÍAADELAMARTÍNEZ ${ }^{5, \mathrm{e}}$, FELIPE POBLETE-VALDERRAMA ${ }^{6, \mathrm{f}}$, \\ CARLOS SALAS ${ }^{7, g}$, CARLOS CRISTI-MONTERO $^{8, \mathrm{~h}}$, \\ CARLOS CELIS-MORALES ${ }^{1,10, j}$
}

\section{The joint effect of sitting time and obesity on the odds of developing type 2 diabetes}

Background: Obesity and sedentary behavior are risk factors acting independently in the development of type 2 diabetes. Aim: To investigate whether the association between diabetes and obesity is modified by the levels of sitting time (ST) in the Chilean population. Material and Methods: We included 4,611 participants from the cross-sectional 2009-2010 Chilean National Health Survey in this study. Diabetes was determined as fasting glucose levels $\geq 126 \mathrm{mg} / \mathrm{dl}$. Body mass index (BMI) and waist circumference (WC) were measured using standardized protocols. Sitting time was assessed using the Global Physical Activity Questionnaire. The association among diabetes, obesity and ST was determined using logistic regression. $\boldsymbol{R e}$ sults: The odds for diabetes increased by 3.1-fold in people with high levels of ST and obesity in comparison to those with low levels of ST and normal BMI (Odds ratio (OR): 4.17 [95\% confidence intervals (CI): 2.87 to 6.05], $P<0.01)$. The odds for diabetes increased by 2.7fold in highly sedentary subjects with central obesity (OR: 3.73 [95\% IC: 2.61 to 5.33], $P<0.01)$ in comparison to those with low levels of ST and normal WC. Conclusions: Elevated levels of sitting time and obesity are associated with a higher odds of developing diabetes.

(Rev Med Chile 2018; 146: 433-441)

Key words: Diabetes Mellitus, Type 2; Obesity; Sedentary Lifestyle.
'BHF Glasgow Cardiovascular Research Centre, Institute of Cardiovascular and Medical Science, University of Glasgow, Glasgow, United Kingdom.

2Escuela de Educación Física, Universidad San

Sebastián, Concepción, Chile.

${ }^{3}$ Grupo de Investigación Calidad de Vida,

Departamento de Ciencias de la Educación,

Universidad del Biobío, Chillán, Chile.

4Instituto de Anatomía, Histología y Patología,

Facultad de Medicina, Universidad Austral de Chile, Valdivia, Chile.

IInstituto de Farmacia, Facultad de Ciencias, Universidad Austral de Chile, Valdivia, Chile.

${ }^{6}$ Escuela de Kinesiología, Facultad de Salud, Universidad Santo Tomás, Sede Valdivia. Chile. ${ }^{7}$ Departamento de Educación Física. Facultad de Educación. Universidad de Concepción. Concepción, Chile.

${ }^{8}$ Grupo IRyS. Escuela de Educación Física. Pontificia Universidad Católica de Valparaíso, Chile.

'Escuela de Kinesiología, Facultad de Salud, Universidad Católica Silva Henríquez, Santiago. Chile.

${ }^{10} \mathrm{Centro}$ de Investigación en Fisiología del Ejercicio (CIFE), Universidad Mayor, Santiago, Chile.

aNutricionista. MSc. Nutrición Humana.

bProfesor de Educación Física, Doctor en Actividad física, Educación Física y Deporte.

'Profesor de Educación Física, MSc Educación.

dProfesora de Biología y Química. MSc.

Neurociencias y Salud Mental.

eBioquímica. MSc. Nutrición y Dietética.

fProfesor de Educación Física, MSc. Educación

en Salud y Bienestar Humano.

gProfesor de Educación Física, MSc Educación

Física.

hProfesor de Educación Física, Doctor en Ciencias de la Actividad Física y del Deporte 'Kinesiólogo. MSc. Educación en Salud y Bienestar Humano.

'Profesor de Educación Física, Doctor en Ciencias Cardiovasculares y Biomédicas.

*FP y AGM contribuyeron igualmente a este manuscrito y son primer autor compartido. Los autores declaran no tener conflictos de interés.

Recibido el 12 de mayo de 2017, aceptado el 3 de abril de 2018.

Correspondencia a:

Dr. Carlos Celis-Morales

BHF Glasgow Cardiovascular Research Centre, 126 University Avenue, Glasgow University,

Glasgow, United Kingdom, G12 8TA.

Tel: 44(0)1413304201

carlos.celis@glasgow.ac.uk

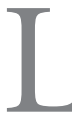
a diabetes mellitus tipo 2 (DMT2) es una enfermedad metabólica progresiva caracterizada por resistencia a la insulina e insuficiencia funcional de las células beta pancreáticas $^{1-3}$. La mayoría de los pacientes con esta forma de diabetes son obesos, y los que no lo son, según los criterios tradicionales de índice de masa corporal (IMC), pueden tener un mayor porcentaje de grasa corporal distribuida predominantemente en la región abdominal ${ }^{2}$. 
Acorde a cifras de la Organización Mundial de Salud (OMS) y de la Federación Internacional de Diabetes (IDF), la prevalencia de DMT2 ha incrementado dramáticamente en las últimas décadas, afectando a 8,5\% de la población mundial en el año 2014 y estimándose que afectará a más de 592 millones de personas en el año 2035 $5^{4-6}$. En Chile, la última Encuesta Nacional de Salud 2016-2017 evidenció que 12,3\% de la población padece esta patología, es decir, 30,9\% más que en $2009-2010^{7,8}$.

Una alimentación saludable, la realización de actividad física (AF) de manera regular y el mantenimiento de un peso corporal normal son parte de las recomendaciones realizadas a todo nivel para prevenir o retrasar la aparición de DMT24; sin embargo, hasta la fecha, pocas campañas se han centrado en la disminución del comportamiento sedentario como un factor clave en la prevención de esta enfermedad.

El término comportamiento sedentario es completamente diferente al término "inactividad física", tal como ha sido reportado por Cristi-Montero et al. ${ }^{9}$. El sedentarismo se define como cualquier comportamiento de vigilia caracterizado por un gasto energético $\leq 1,5$ METs (metabolic-energy-equivalents), como ver televisión o estar sentado mientras se trabaja ${ }^{10}$, estimándose que un adulto pasa entre 55 y 70\% del día en actividades que involucran comportamientos sedentarios ${ }^{10,11}$. Estudios anteriores han evidenciado que el comportamiento sedentario es un factor de riesgo en el desarrollo de DMT2 y síndrome metabólico, independiente de los niveles de $\mathrm{AF}^{12-14}$. Considerando que, en promedio, $35,9 \%$ de la población destina más de $4 \mathrm{~h}$ al día a actividades de tipo sedentarias ${ }^{15} \mathrm{y}$ que la IDF ha estimado que cada seis segundos una persona muere por DMT2 $2^{5,16}$, el objetivo de este estudio fue investigar si la asociación entre DMT2 y obesidad son modificadas por los niveles de sedentarismo en población chilena.

\section{Materiales y Métodos}

\section{Diseño del estudio}

La muestra seleccionada comprende a los participantes de la Encuesta Nacional de Salud 2009-2010 (ENS 2009-2010) ${ }^{7}$. La ENS 2009-2010 corresponde a un estudio de prevalencia realizado en hogares en una muestra nacional, probabilística, estratificada y multietápica de 5.412 personas mayores de 15 años con representatividad nacional, regional, y área urbana/rural. En este estudio fueron incluidos un total de 4.611 participantes mayores de 18 años, que tenían información disponible en relación a su tiempo sedente y niveles de obesidad. Se excluyeron de los análisis a personas clasificadas con bajo peso corporal según IMC (IMC $\left.<18,5 \mathrm{~kg} / \mathrm{m}^{2}\right)$. El protocolo de estudio fue aprobado por el Comité de Ética de la Escuela de Medicina de la Pontificia Universidad Católica de Chile. Todos los participantes firmaron un consentimiento informado ${ }^{7}$.

\section{Mediciones metabólicas y antropométricas}

La glicemia basal fue medida a través de una muestra de sangre en ayuno con métodos estandarizados y previamente descritos en la ENS 2009$2010^{7}$. Para la detección de DMT2 se consideró una glicemia en ayuno $\geq 126 \mathrm{mg} / \mathrm{dl}$ o el autorreporte de diagnóstico médico de DMT2. Se clasificó el estado nutricional según IMC en base a las recomendaciones de la OMS: normo peso: $18,5-24,9 \mathrm{~kg} / \mathrm{m}^{2}$; sobrepeso: $25,0-29,9 \mathrm{~kg} / \mathrm{m}^{2}$ y obesidad $\geq 30,0 \mathrm{~kg} /$ $\mathrm{m}^{2}{ }^{17}$, mientras que la obesidad central fue definida por un perímetro de cintura $(\mathrm{PC}) \geq 83 \mathrm{~cm}$ para mujeres $\mathrm{y} \geq 88 \mathrm{~cm}$ para hombres, según los puntos de corte sugeridos por la ENS 2009-20107.

Las variables sociodemográficas incluidas fueron edad, sexo, zona geográfica, nivel educacional, e ingreso económico. Los datos asociados con estilos de vida, autorreporte de salud y bienestar, tabaquismo y consumo de frutas, verduras, alcohol y sal, se obtuvieron mediante la aplicación de cuestionarios validados en la ENS 2009-2010².

\section{Clasificación de actividad física}

Los niveles de AF, el tiempo destinado a las actividades de transporte, y las actividades de intensidad moderada o vigorosa de la población, fueron determinados con el cuestionario "Global Physical Activity Questionnaire" (GPAQ v2) ${ }^{18}$, el cual ha sido validado internacionalmente ${ }^{19} \mathrm{y}$ en población latina ${ }^{20}$. La AF total es presentada como la suma del tiempo reportado en actividades de transporte, de intensidad moderada y vigorosa, tanto en el trabajo como en el tiempo libre. Esta variable fue expresada en MET/min/semana, para considerar las diferencias en gasto energético asociada a cada una de las actividades. Se consi- 
deró como punto de corte para inactividad física un gasto energético menor a $600 \mathrm{METs} / \mathrm{min} /$ semana $^{18,21}$. Los niveles de sedentarismo fueron determinados mediante el autorreporte de tiempo destinado a actividades que involucren estar sentado o reclinado durante el tiempo libre o de trabajo (por ejemplo, tiempo sentado frente al computador o TV, viajando en bus, tren o auto) a través del mismo cuestionario GPAQ v2. Se consideró como un alto nivel de sedentarismo cuando una persona destinaba más de $4 \mathrm{~h}$ a estar sentada al día y bajo nivel cuando destinaba menos de 4 h al día ${ }^{15}$.

\section{Quintiles de IMC y PC}

Los puntos de corte para quintiles de IMC fueron Q1: $18,5-19,9 \mathrm{~kg} / \mathrm{m}^{2}$; Q2: 20,0 a $24,9 \mathrm{~kg} /$ $\mathrm{m}^{2}$; Q3: 25,0 a $29,9 \mathrm{~kg} / \mathrm{m}^{2}$; Q4: 30,0 a $34,9 \mathrm{~kg} / \mathrm{m}^{2}$; $\mathrm{Q} 5: \geq 35,0 \mathrm{~kg} / \mathrm{m}^{2}$. Los puntos de corte para quintiles de PC en mujeres fueron Q1: $<86,0 \mathrm{~cm}$; Q2: 86,1 a $92 \mathrm{~cm}$; Q3: 92,1 a $98 \mathrm{~cm}$; Q4: 98,1 a $107,0 \mathrm{~cm}$; Q5: $>107,0 \mathrm{~cm}$ y para quintiles de PC en hombres Q1: < 87,0 cm; Q2: 87,1 a 93,0 cm; Q3: 93,1 a 98,0 cm; Q4: 98,1 a 105,0 cm; Q5: > 105,0 cm.

\section{Análisis estadístico}

Los datos de caracterización de la población estudiada son presentados como promedio y desviación estándar (DE) para variables continuas, y como porcentaje para variables categóricas.

La asociación entre DMT2 con los niveles de sedentarismo (bajos o altos) y obesidad (estado nutricional normal u obesidad) fue investigada mediante análisis de regresión logística y reportada como Odds Ratio (OR) y con un intervalo de confianza del 95\% (OR 95\% IC), utilizando como grupo de referencias aquellos participantes clasificados con bajos niveles de sedentarismo y con un IMC o PC normal.

La asociación entre DMT2 y quintiles de IMC o PC según niveles de sedentarismo fue investigada mediante análisis de regresión logística utilizando como grupo de referencia aquellos participantes clasificados con bajos niveles de sedentarismo y que se encontraban en el quintil más bajo para IMC o PC (Quintil 1). Los análisis fueron ajustados por edad, sexo, educación, tabaquismo, AF y consumo de frutas y verduras. Para todos los análisis se utilizó el módulo de muestras complejas del programa STATA SE v14 y fueron estimados utilizando muestras expandidas según la ENS 2009-20107. El nivel de significancia fue definido como $\mathrm{p}<0,05$.

\section{Resultados}

Las características de los participantes, según las categorías derivadas de los niveles de sedentarismo y obesidad, se presentan en la Tabla 1, las características según los niveles de sedentarismo y obesidad central en la Tabla 2. En general, las personas clasificadas con bajos niveles de sedentarismo y con un IMC normal tenían una menor edad, realizaban una mayor cantidad de AF total y, por ende, presentaron una menor prevalencia de inactividad física; a su vez, consumían una menor cantidad de frutas, verduras y sal, y poseían un menor porcentaje de complicaciones metabólicas comparadas con las personas altamente sedentarias y obesas (Tabla 1). Características similares fueron observadas al analizar los niveles de sedentarismo y obesidad central (Tabla 2).

La Figura 1 presenta la asociación entre DMT2 con los niveles de sedentarismo y obesidad. Como se aprecia en la Figura 1A, en personas con bajos niveles de sedentarismo y con obesidad, el riesgo de DMT2 aumenta 200\% en comparación a aquellas con bajos niveles de sedentarismo, pero con IMC normal (OR: 3,02 [95\% IC: 2,10 a 4,34], $\mathrm{p}<0,0001)$. No se observó riesgo significativo en individuos que presentaban altos niveles de sedentarismo, pero un IMC normal. Sin embargo, el riesgo de DMT2 aumentó 317\% en personas con un alto nivel de sedentarismo y con obesidad, en comparación a aquellos con un bajo nivel de sedentarismo y con IMC normal (OR: 4,17 [95\% IC: 2,87 a 6,05], $\mathrm{p}<0,0001$ ).

Al utilizar el PC como indicador de obesidad central, el riesgo de DMT2 fue menor que el observado para obesidad según IMC (Figura 1B). Al comparar sujetos con bajos niveles de sedentarismo y sin obesidad central con sujetos con bajos niveles de sedentarismo y con obesidad central, el riesgo de DMT2 incrementó 169\% (OR: 2,69 [95\% IC: 1,90 a 3,82], $\mathrm{p}<0,0001$ ); no obstante, el riesgo aumentó $273 \%$ en sujetos altamente sedentarios y con obesidad central (OR: 3,73 [95\% IC: 2,61 a 5,33], $\mathrm{p}<0,0001)$.

$\mathrm{Al}$ investigar la asociación entre quintiles de IMC o PC, tiempo destinado a estar sentado y el 
Tabla 1. Características de la población chilena según obesidad y niveles de tiempo destinado a estar sentado

\begin{tabular}{|c|c|c|c|c|c|}
\hline & $\begin{array}{c}\text { Bajos niveles } \\
\text { de sedenta- } \\
\text { rismo e IMC } \\
\text { normal* }\end{array}$ & $\begin{array}{l}\text { Bajos niveles } \\
\text { de sedenta- } \\
\text { rismo e IMC } \\
\geq 30,0 \mathrm{~kg} / \mathrm{m}^{2}\end{array}$ & $\begin{array}{c}\text { Altos niveles } \\
\text { de sedenta- } \\
\text { rismo e IMC } \\
\text { normal }\end{array}$ & $\begin{array}{l}\text { Altos niveles } \\
\text { de sedenta- } \\
\text { rismo e IMC } \\
\geq 30,0 \mathrm{~kg} / \mathrm{m}^{2}\end{array}$ & $\begin{array}{c}\text { Valor } \\
\text { p }\end{array}$ \\
\hline $\begin{array}{l}\text { Sociodemográficas } \\
\text { n } \\
\text { Mujeres (\%) } \\
\text { Edad (años) }\end{array}$ & $\begin{array}{c}781 \\
56,2 \\
41,9 \pm 18,8\end{array}$ & $\begin{array}{c}796 \\
73,5 \\
49,6 \pm 15,1^{*}\end{array}$ & $\begin{array}{c}547 \\
62,0 \\
39,1 \pm 21,3^{*}\end{array}$ & $\begin{array}{c}535 \\
60,0 \\
51,1 \pm 18,3^{*}\end{array}$ & $\begin{array}{l}<0,0001 \\
<0,0001\end{array}$ \\
\hline $\begin{array}{l}\text { Zona geográfica (\%) } \\
\text { Rural } \\
\text { Urbana }\end{array}$ & $\begin{array}{l}16,8 \\
83,2\end{array}$ & $\begin{array}{l}21,0 \\
79,0\end{array}$ & $\begin{array}{c}7,3 \\
92,7\end{array}$ & $\begin{array}{l}10,8 \\
89,2\end{array}$ & $<0,0001$ \\
\hline $\begin{array}{l}\text { Grupo etario (\%) } \\
<25 \text { años } \\
25-44 \text { años } \\
45-64 \text { años } \\
\geq 65 \text { años }\end{array}$ & $\begin{array}{l}22,5 \\
35,9 \\
27,4 \\
14,2\end{array}$ & $\begin{array}{l}4,8 \\
32,3 \\
44,9 \\
18,0\end{array}$ & $\begin{array}{l}34,9 \\
31,1 \\
17,0 \\
17,0\end{array}$ & $\begin{array}{l}7,8 \\
31,4 \\
33,5 \\
27,3\end{array}$ & $<0,0001$ \\
\hline $\begin{array}{l}\text { Nivel educacional (\%) } \\
\text { Básica } \\
\text { Media } \\
\text { Técnico/Universitaria }\end{array}$ & $\begin{array}{l}23,3 \\
59,3 \\
17,4\end{array}$ & $\begin{array}{l}34,5 \\
54,7 \\
10,8\end{array}$ & $\begin{array}{l}14,4 \\
54,0 \\
31,6\end{array}$ & $\begin{array}{l}30,3 \\
51,5 \\
18,2\end{array}$ & $<0,0001$ \\
\hline $\begin{array}{l}\text { Nivel de ingreso (\%) } \\
\text { Bajo } \\
\text { Medio } \\
\text { Alto }\end{array}$ & $\begin{array}{r}59,4 \\
30,7 \\
9,9\end{array}$ & $\begin{array}{r}64,9 \\
28,7 \\
6,4\end{array}$ & $\begin{array}{l}47,3 \\
35,0 \\
17,7\end{array}$ & $\begin{array}{l}49,9 \\
36,6 \\
13,5\end{array}$ & $<0,0001$ \\
\hline $\begin{array}{l}\text { Antropométricas } \\
\text { Peso corporal }(\mathrm{kg}) \\
\text { IMC }\left(\mathrm{kg} / \mathrm{m}^{2}\right) \\
\text { Perímetro de cintura }(\mathrm{cm}) \\
\text { Obesidad central }(\%)\end{array}$ & $\begin{array}{c}59,6 \pm 8,3 \\
22,8 \pm 1,7 \\
86,6 \pm 7,9 \\
23,0\end{array}$ & $\begin{array}{c}84,2 \pm 12,1^{*} \\
34,0 \pm 4,3^{*} \\
107,4 \pm 10,1^{*} \\
99,2\end{array}$ & $\begin{array}{c}58,7 \pm 7,8 \\
22,5 \pm 1,7 \\
86,6 \pm 9,8 \\
22,9\end{array}$ & $\begin{array}{c}88,5 \pm 14,3^{*} \\
34,6 \pm 4,8^{*} \\
109,7 \pm 11,4^{*} \\
99,6\end{array}$ & $\begin{array}{l}<0,0001 \\
<0,0001 \\
<0,0001 \\
<0,0001\end{array}$ \\
\hline $\begin{array}{l}\text { Estilo de vida } \\
\text { Actividad física total (MET/h/semana) } \\
\text { Actividad física de transporte (min/día) } \\
\text { Actividad física moderada (min/día) } \\
\text { Actividad física vigorosa (min/día) } \\
\text { Prevalencia inactividad física (\%) } \\
\text { Tiempo sedente (h/día) } \\
\text { Consumo de frutas y vegetales (g/día) } \\
\text { Consumo de sal (g/día) } \\
\text { Consumo de alcohol (g/día) }\end{array}$ & $\begin{array}{c}140,2 \pm 157,1 \\
50,4 \pm 78,9 \\
117,3 \pm 153,7 \\
66,4 \pm 139,6 \\
16,3 \\
1,7 \pm 0,8 \\
207,2 \pm 132,1 \\
9,2 \pm 2,1 \\
51,3 \pm 59,4\end{array}$ & $\begin{array}{c}134,6 \pm 147,5 \\
52,1 \pm 86,0 \\
124,6 \pm 159,5 \\
55,9 \pm 122,1 \\
20,9 \\
1,7 \pm 0,8 \\
216,1 \pm 135,4 \\
10,2 \pm 2,5^{\star} \\
60,8 \pm 134,2\end{array}$ & $\begin{array}{c}90,2 \pm 124,1^{*} \\
56,7 \pm 108,1 \\
73,3 \pm 121,5^{*} \\
31,6 \pm 93,6^{*} \\
29,3 \\
6,1 \pm 2,3^{*} \\
212,9 \pm 141,2 \\
8,5 \pm 2,0^{*} \\
51,6 \pm 60,5\end{array}$ & $\begin{array}{c}90,1 \pm 131,9^{*} \\
41,8 \pm 84,0 \\
83,7 \pm 138,6^{*} \\
33,8 \pm 106,2^{*} \\
39,1 \\
6,0 \pm 2,3^{*} \\
229,3 \pm 143,3^{*} \\
10,3 \pm 2,5^{*} \\
59,9 \pm 93,2\end{array}$ & $\begin{aligned}< & 0,0001 \\
& 0,249 \\
< & 0,0001 \\
< & 0,0001 \\
< & 0,0001 \\
< & 0,0001 \\
& 0,018 \\
& 0,001 \\
& 0,564\end{aligned}$ \\
\hline $\begin{array}{l}\text { Autoreporte salud y bienestar (\%) } \\
\text { Malo } \\
\text { Regular } \\
\text { Bueno }\end{array}$ & $\begin{array}{l}2,3 \\
31,4 \\
66,3\end{array}$ & $\begin{array}{l}3,5 \\
37,8 \\
58,7\end{array}$ & $\begin{array}{l}3,3 \\
24,3 \\
72,4\end{array}$ & $\begin{array}{c}4,1 \\
32,5 \\
63,4\end{array}$ & $<0,0001$ \\
\hline $\begin{array}{l}\text { Tabaquismo (\%) } \\
\text { Nunca } \\
\text { Exfumador } \\
\text { Fumador }\end{array}$ & $\begin{array}{l}42,4 \\
18,8 \\
38,8\end{array}$ & $\begin{array}{l}42,0 \\
27,4 \\
30,6\end{array}$ & $\begin{array}{l}42,8 \\
17,7 \\
39,5\end{array}$ & $\begin{array}{l}40,6 \\
29,3 \\
30,1\end{array}$ & $<0,0001$ \\
\hline $\begin{array}{l}\text { Metabólicas } \\
\text { Síndrome metabólico } \\
\text { DMT2 } \\
\text { Hipertensión arterial } \\
\text { PAS }(\mathrm{mmHg}) \\
\text { PAD }(\mathrm{mmHg})\end{array}$ & $\begin{array}{c}8,8 \\
5,9 \\
19,7 \\
120,6 \pm 20,5 \\
71,5 \pm 10,4\end{array}$ & $\begin{array}{c}60,1 \\
15,9 \\
43,4 \\
132,8 \pm 22,2^{*} \\
79,9 \pm 10,8^{*}\end{array}$ & $\begin{array}{c}7,2 \\
6,1 \\
17,7 \\
119,6 \pm 20,5 \\
71,4 \pm 10,0\end{array}$ & $\begin{array}{c}67,5 \\
20,6 \\
45,6 \\
134,1 \pm 22,2^{*} \\
79,7 \pm 11,0^{*}\end{array}$ & $\begin{array}{l}<0,0001 \\
<0,0001 \\
<0,0001 \\
<0,0001 \\
<0,0001\end{array}$ \\
\hline
\end{tabular}

Datos presentados como promedio y desviación estándar (DE) para variables continuas y como porcentaje para variables categóricas. Se consideró como punto de corte para altos niveles de sedentarismo $\geq 4 \mathrm{~h}$ al día de tiempo destinado a estar sentado. Se consideró IMC normal: 18,5-25,0 kg/m² y obesidad: IMC $\geq 30,0 \mathrm{~kg} / \mathrm{m}^{2}$. Diferencias estadísticamente significativas entre las categorías de sedentarismo e IMC, para variables continuas, fueron determinadas con análisis de regresión lineal y con el test de Chi-cuadrado para variables categóricas. Para variables continuas, se ocupó el grupo con bajos niveles de sedentarismo y IMC normal como referencia, por ende * indica si las diferencias entre este grupo y las otras categorías fueron estadísticamente significativas $(p<0,05)$. PAS: presión arterial sistólica. PAD: presión arterial diastólica. 
Tabla 2. Características de la población chilena según obesidad central y niveles de tiempo destinado a estar sentado

\begin{tabular}{|c|c|c|c|c|c|}
\hline & $\begin{array}{l}\text { Bajos niveles de } \\
\text { sedentarismo } \\
\text { y sin obesidad } \\
\text { central* }\end{array}$ & $\begin{array}{l}\text { Bajos niveles de } \\
\text { sedentarismo y } \\
\text { obesidad central }\end{array}$ & $\begin{array}{l}\text { Altos niveles de } \\
\text { sedentarismo } \\
\text { y sin obesidad } \\
\text { central }\end{array}$ & $\begin{array}{l}\text { Altos niveles de } \\
\text { sedentarismo y } \\
\text { obesidad central }\end{array}$ & $\begin{array}{c}\text { Valor } \\
\text { p }\end{array}$ \\
\hline $\begin{array}{l}\text { Sociodemográficas } \\
\text { n } \\
\text { Mujeres (\%) } \\
\text { Edad (años) }\end{array}$ & $\begin{array}{c}833 \\
59,3 \\
39,6 \pm 18,1\end{array}$ & $\begin{array}{c}1.961 \\
62,0 \\
49,8 \pm 15,7^{*}\end{array}$ & $\begin{array}{c}579 \\
64,4 \\
35,1 \pm 19,3^{*}\end{array}$ & $\begin{array}{c}1.238 \\
54,4 \\
50,2 \pm 18,9^{*}\end{array}$ & $\begin{array}{l}<0,0001 \\
<0,0001\end{array}$ \\
\hline $\begin{array}{l}\text { Zona geográfica(\%) } \\
\text { Rural } \\
\text { Urbana }\end{array}$ & $\begin{array}{l}16,1 \\
83,9\end{array}$ & $\begin{array}{l}19,2 \\
80,8\end{array}$ & $\begin{array}{l}7,6 \\
92,4\end{array}$ & $\begin{array}{c}9,9 \\
90,1\end{array}$ & $<0,0001$ \\
\hline $\begin{array}{l}\text { Grupo etario (\%) } \\
<25 \text { años } \\
25-44 \text { años } \\
45-64 \text { años } \\
\geq 65 \text { años }\end{array}$ & $\begin{array}{l}26,3 \\
37,8 \\
24,5 \\
11,4\end{array}$ & $\begin{array}{l}5,4 \\
32,0 \\
43,2 \\
19,4\end{array}$ & $\begin{array}{l}41,8 \\
32,0 \\
14,2 \\
12,0\end{array}$ & $\begin{array}{r}9,8 \\
32,9 \\
31,7 \\
25,6\end{array}$ & $<0,0001$ \\
\hline $\begin{array}{l}\text { Nivel educacional (\%) } \\
\text { Básica } \\
\text { Media } \\
\text { Técnico/Universitaria }\end{array}$ & $\begin{array}{l}19,6 \\
62,1 \\
18,3\end{array}$ & $\begin{array}{l}31,9 \\
54,7 \\
13,4\end{array}$ & $\begin{array}{l}11,5 \\
57,2 \\
31,3\end{array}$ & $\begin{array}{l}28,4 \\
48,5 \\
23,1\end{array}$ & $<0,0001$ \\
\hline $\begin{array}{l}\text { Nivel de ingreso (\%) } \\
\text { Bajo } \\
\text { Medio } \\
\text { Alto }\end{array}$ & $\begin{array}{l}56,6 \\
33,3 \\
10,1\end{array}$ & $\begin{array}{c}62,4 \\
29,6 \\
8,0\end{array}$ & $\begin{array}{l}47,7 \\
36,6 \\
15,7\end{array}$ & $\begin{array}{l}47,5 \\
37,5 \\
15,0\end{array}$ & $<0,0001$ \\
\hline $\begin{array}{l}\text { Antropométricas } \\
\text { Peso corporal }(\mathrm{kg}) \\
\text { IMC }\left(\mathrm{kg} / \mathrm{m}^{2}\right)\end{array}$ & $\begin{array}{l}59,4 \pm 8,6 \\
23,2 \pm 2,6\end{array}$ & $\begin{array}{c}76,3 \pm 12,4^{*} \\
29,9 \pm 4,7^{*}\end{array}$ & $\begin{array}{l}58,9 \pm 8,8 \\
22,7 \pm 2,7\end{array}$ & $\begin{array}{c}78,9 \pm 15,1^{*} \\
30,2 \pm 5,2^{*}\end{array}$ & $\begin{array}{l}<0,0001 \\
<0,0001\end{array}$ \\
\hline $\begin{array}{l}\text { Estado nutricional (\%) } \\
18,5-24,9 \mathrm{~kg} / \mathrm{m}^{2} \\
25,0-29,9 \mathrm{~kg} / \mathrm{m}^{2} \\
\geq 30,0 \mathrm{~kg} / \mathrm{m}^{2} \\
\text { Perímetro de cintura }(\mathrm{cm})\end{array}$ & $\begin{array}{c}75,9 \\
23,4 \\
0,7 \\
85,7 \pm 8,3\end{array}$ & $\begin{array}{c}9,2 \\
50,2 \\
40,6 \\
100,9 \pm 9,8^{*}\end{array}$ & $\begin{array}{c}78,7 \\
21,0 \\
0,3 \\
84,9 \pm 9,5\end{array}$ & $\begin{array}{c}10,2 \\
46,4 \\
43,4 \\
102,4 \pm 11,3^{*}\end{array}$ & $\begin{array}{l}<0,0001 \\
<0,0001\end{array}$ \\
\hline $\begin{array}{l}\text { Estilo de vida } \\
\text { Actividad física total (MET/h/semana) } \\
\text { Actividad física de transporte (min/día) } \\
\text { Actividad física moderada (min/día) } \\
\text { Actividad física vigorosa (min/día) } \\
\text { Prevalencia inactividad física (\%) } \\
\text { Tiempo sedente (h/día) } \\
\text { Consumo de frutas y vegetales (g/día) } \\
\text { Consumo de sal (g/día) } \\
\text { Consumo de alcohol (g/día) }\end{array}$ & $\begin{array}{c}147,1 \pm 158,4 \\
54,0 \pm 83,9 \\
120,4 \pm 156,6 \\
70,4 \pm 141,5 \\
14,5 \\
1,7 \pm 0,8 \\
208,0 \pm 130,5 \\
9,1 \pm 2,2 \\
48,6 \pm 56,4\end{array}$ & $\begin{array}{c}135,6 \pm 149,1^{*} \\
49,1 \pm 81,1 \\
124,9 \pm 159,0 \\
58,2 \pm 128,4^{*} \\
18,8 \\
1,7 \pm 0,9 \\
216,2 \pm 138,5 \\
10,1 \pm 2,6 \\
53,9 \pm 105,8\end{array}$ & $\begin{array}{c}91,1 \pm 125,9^{*} \\
58,8 \pm 101,8 \\
66,4 \pm 115,4^{*} \\
35,0 \pm 103,6^{*} \\
25,6 \\
6,0 \pm 2,2^{*} \\
206,7 \pm 135,8 \\
8,7 \pm 2,6 \\
51,4 \pm 61,7\end{array}$ & $\begin{array}{c}85,0 \pm 122,6^{*} \\
42,1 \pm 84,4^{*} \\
80,8 \pm 138,3^{*} \\
29,6 \pm 94,2^{*} \\
36,4 \\
6,1 \pm 2,4^{*} \\
225,8 \pm 145,3^{*} \\
9,9 \pm 2,9 \\
53,8 \pm 84,3\end{array}$ & $\begin{aligned}< & 0,0001 \\
& 0,009 \\
< & 0,0001 \\
< & 0,0001 \\
< & 0,0001 \\
< & 0,0001 \\
& 0,019 \\
& 0,193 \\
& 0,596\end{aligned}$ \\
\hline $\begin{array}{l}\text { Autoreporte salud y bienestar (\%) } \\
\text { Malo } \\
\text { Regular } \\
\text { Bueno }\end{array}$ & $\begin{array}{l}1,8 \\
29,1 \\
69,1\end{array}$ & $\begin{array}{l}3,0 \\
34,7 \\
62,3\end{array}$ & $\begin{array}{r}3,8 \\
21,4 \\
74,8\end{array}$ & $\begin{array}{c}3,7 \\
30,7 \\
65,6\end{array}$ & $<0,0001$ \\
\hline $\begin{array}{l}\text { Tabaquismo (\%) } \\
\text { Nunca } \\
\text { Exfumador } \\
\text { Fumador }\end{array}$ & $\begin{array}{l}42,9 \\
18,6 \\
38,5\end{array}$ & $\begin{array}{l}41,1 \\
25,4 \\
33,5\end{array}$ & $\begin{array}{l}44,6 \\
16,9 \\
38,5\end{array}$ & $\begin{array}{l}38,0 \\
28,4 \\
33,6\end{array}$ & $<0,0001$ \\
\hline $\begin{array}{l}\text { Metabólicas } \\
\text { Síndrome metabólico } \\
\text { DMT2 } \\
\text { Hipertensión arterial } \\
\text { PAS }(\mathrm{mmHg}) \\
\text { PAD }(\mathrm{mmHg})\end{array}$ & $\begin{array}{c}3,9 \\
4,9 \\
16,6 \\
119,0 \pm 20,2 \\
71,1 \pm 10,2\end{array}$ & $\begin{array}{c}49,9 \\
12,1 \\
37,1 \\
131,0 \pm 21,9^{*} \\
78,4 \pm 10,7^{*}\end{array}$ & $\begin{array}{c}2,1 \\
5,1 \\
12,9 \\
117,4 \pm 19,1 \\
70,6 \pm 9,6\end{array}$ & $\begin{array}{c}53,9 \\
16,0 \\
39,7 \\
131,6 \pm 22,9^{*} \\
77,9 \pm 11,3^{*}\end{array}$ & $\begin{array}{l}<0,0001 \\
<0,0001 \\
<0,0001 \\
<0,0001 \\
<0,0001\end{array}$ \\
\hline
\end{tabular}

Datos presentados como promedio y desviación estándar (DE) para variables continuas y como porcentaje para variables categóricas. Se consideró como punto de corte para altos niveles de sedentarismo $\geq 4 \mathrm{~h}$ al día de tiempo destinado a estar sentado. Para obesidad central se consideró un PC (PC $\geq 83 \mathrm{~cm}$ y $\geq 88 \mathrm{~cm}$ para mujeres y hombres, respectivamente). Diferencias entre las categorías de sedentarismo e IMC, para variables continuas, fueron determinadas con análisis de regresión lineal y con el test de Chi-cuadrado para variables categóricas. Para variables continuas, se ocupó el grupo son bajos niveles de sedentarismo y IMC normal como grupo de referencia, por ende * indica si las diferencias entre los otros grupos y este grupo de referencia fueron estadísticamente significativas $(p<0,05)$. PAS: presión arterial sistólica. PAD: presión arterial diastólica. 
riesgo de diabetes según niveles de sedentarismo, se identificó una interacción significativa a incrementar el riesgo de DMT2 (Figura 2). El riesgo de diabetes mellitus tipo 2 incrementó en $257 \%$ en personas con bajos niveles de sedentarismo y que se encontraba en el quintil más alto de IMC (OR: 3,57 [95\% IC: 2,31 a 5,53], p < 0,0001) en comparación a personas con bajos niveles de sedentarismo que se encontraban en quintil más bajo de IMC. De igual manera, los individuos

\section{A}

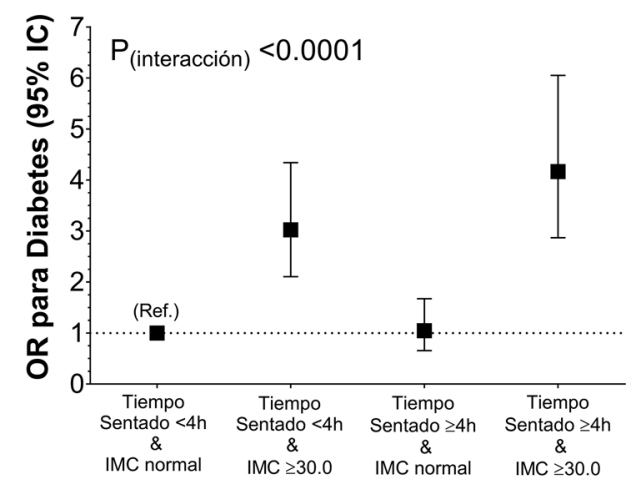

B

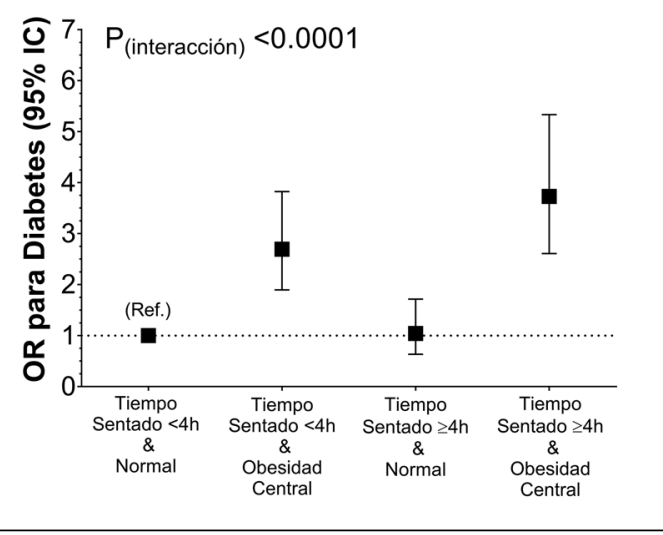

Figura 1. Riesgo de diabetes mellitus tipo 2 según indicadores de obesidad y tiempo destinado a estar sentado. Datos presentados como odds ratio (95\% IC) para diabetes mellitus tipo 2 según nivel de obesidad (IMC y PC y tiempo destinado a estar sentado). La Figura A utilizó IMC $\geq 30,0 \mathrm{~kg} / \mathrm{m}^{2}$ y la Figura B utilizó obesidad central ( $\geq 83 \mathrm{~cm}$ para mujeres y $\geq 88 \mathrm{~cm}$ para hombres) y tiempo destinado a estar sentado. Se utilizó como grupo de referencia para los análisis aquellas personas clasificadas como tiempo destinado a estar sentado $<4 \mathrm{~h}$ al día y que se encontraban con un IMC $\left(<25,0 \mathrm{~kg} / \mathrm{m}^{2}\right)$ o PC normal $(<83 \mathrm{~cm}$ para mujeres $\mathrm{y}<88 \mathrm{~cm}$ para hombres). Los análisis fueron ajustados por edad, sexo, educación, tabaquismo, actividad física y consumo de frutas y verduras.

\section{A}

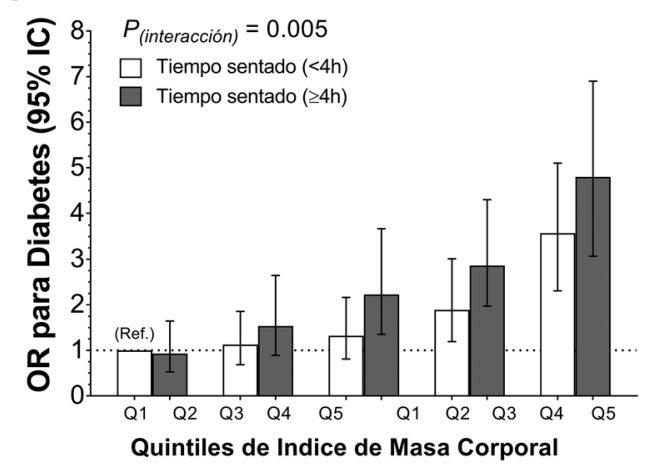

B

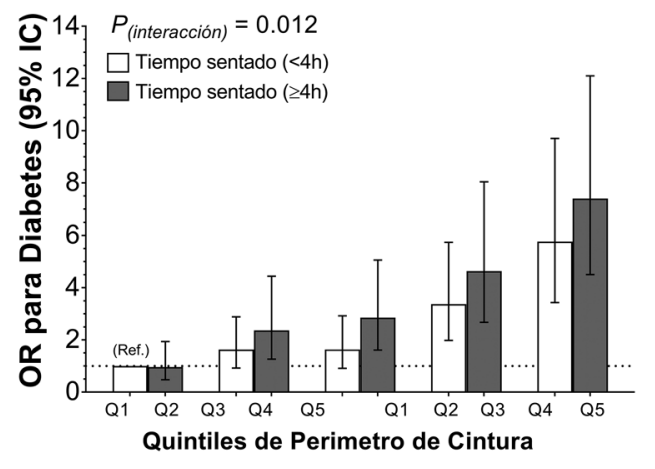

Figura 2. Riesgo de diabetes mellitus tipo 2 para quintiles de IMC (figura 2A) y quintiles de PC (figura 2B) según tiempo destinado a estar sentado. Datos presentados como odds ratio $(95 \%$ IC) para diabetes tipo 2 según quintiles de IMC (Figura $A$ ) y PC (Figura B). Se utilizó como grupo de referencia para los análisis aquellas personas clasificadas como $<4 \mathrm{~h}$ al día de tiempo sentado y que se encontraban en el quintil 1 para IMC o perímetro de cintura (Ref.). Los análisis fueron ajustados por edad, sexo, educación, tabaquismo, actividad física y consumo de frutas y verduras. Los puntos de corte para quintiles de IMC (kg/ $\mathrm{m}^{2}$ ) fueron Q1: 18,5-19,9 (kg/m²); Q2: 20,0 a 24,9; Q3: 25,0 a 29,9; Q4: 30,0 a 34,9; Q5: $\geq 35,0$. Los puntos de corte para quintiles de PC (cm) en mujeres fueron Q1: < 86,0; Q2: 86,1 a 92,0; Q3: 92,1 a 98,0; Q4: 98,1 a 107,0; Q5: > 107,0 y para quintiles de PC (cm) en hombres Q1:< 87,0; Q2: 87,1 a 93,0; Q3: 93,1 a 98,0; Q4: 98,1 a 105,0; Q5: > 105,0. 
altamente sedentarios y que, a su vez, se ubicaban en el quintil más alto de IMC, incrementaron su riesgo de DMT2 en 380\% (OR: 4,80 [95\% IC: 3,07 a 7,52], p <0,0001).

$\mathrm{Al}$ analizar los quintiles de $\mathrm{PC}$ según niveles de sedentarismo, el incremento del riesgo de DMT2 fue mayor que lo observado en los quintiles de IMC (Figura 2B). Las personas con bajos niveles de sedentarismo ubicadas en el quintil más alto de PC tuvieron $477 \%$ de riesgo de DMT2 (OR: 5,77 [95\% IC: 3,43 a 9,70], $\mathrm{p}<0,0001$ ). Por otro lado, las personas altamente sedentarias y en el quintil más alto de $\mathrm{PC}$ incrementaron su riesgo 591\% (OR: 6,91 [95\% IC: 4,08 a 11,8], p < 0,0001) en comparación a personas con bajos niveles de sedentarismo y en el quintil más bajo de PC.

\section{Discusión}

Desde el estudio pionero realizado en 1953 por Morris et al., donde se determinó que los trabajadores que pasaban gran parte de su día sentados presentaban mayor riesgo de eventos cardiovasculares ${ }^{22}$, el estudio de los comportamientos sedentarios y su asociación con el desarrollo de diferentes enfermedades ha sido de gran interés para la comunidad científica.

En este trabajo se logró identificar que presentar altos niveles de sedentarismo y obesidad (ya sea por un elevado IMC o PC) se asocia a un importante incremento en el riesgo de desarrollar DMT2, en comparación a aquellos individuos que presentan bajos niveles de sedentarismo y con un IMC o PC normal. Esta asociación fue independiente de factores de confusión de tipo sociodemográficos y de estilo de vida (tabaquismo, alimentación y AF). De igual manera, las personas con altos niveles de sedentarismo, pero con IMC o PC normal, tienen una menor probabilidad de desarrollar DMT2 que aquellas personas que presentan bajos niveles de sedentarismo, pero son obesas.

Rockette-Wagner et al. determinaron en un grupo de personas con alto riesgo de DMT2, que el riesgo de desarrollar esta patología aumenta en $3,4 \%$ por cada hora que se ve televisión ${ }^{14}$. En Brasil, Turi et al. reportaron que adultos que ven televisión de manera habitual aumentan en 44,7\% el riesgo de mortalidad prematura, independiente de otros posibles factores de confusión y de los niveles de AF. En dicho estudio, la DMT2 fue la mayor causa asociada a mortalidad ${ }^{23}$. En el año 2016, el estudio Maastricht, realizado en 2.497 personas que presentaban, ya sea glicemia normal, alterada o con diagnóstico de DMT2, identificó que cada hora extra de tiempo sedentario se asociaba con un aumento de $22 \%$ de la probabilidad de desarrollar DMT2 ${ }^{24}$.

Los hallazgos de nuestro estudio coinciden con estos datos, pudiendo ser de gran utilidad a nivel de salud pública vinculados a las estrategias que se están realizando y se deberían realizar para la prevención de DMT2 en nuestro país. Si consideramos que en el año 2010 el gasto mundial en pacientes con DMT2 alcanzó a 376 billones de dólares, estimándose que el gasto en Chile fue de aproximadamente 571 dólares por persona ${ }^{25}$; que las personas poseen mayoritariamente un trabajo de tipo sedente, en el cual están al menos $8 \mathrm{~h}$ al día sentadas; y que cerca de $46 \%$ de los casos de DMT2 aún no han sido diagnosticados ${ }^{5}$, deberíamos reflexionar que la tarea por realizar aún es amplia y compleja.

Sin duda han sido descritos en la literatura los grandes beneficios de la realización de AF de manera regular como factor protector del desarrollo de DMT2 o sus complicaciones en personas que ya la padecen; no obstante, un gran porcentaje de la población es inactiva, por lo tanto, es posible que programas destinados a reducir el tiempo sedentario podrían ser una estrategia efectiva, fácil de realizar y con un alto impacto en la salud de la población. En este contexto, futuras intervenciones deberían centrarse no solo en la promoción de la AF de intensidad moderada o vigorosa, sino también deberían contemplar las acciones dirigidas a disminuir el tiempo sedente.

Entre las limitaciones de este estudio se encuentra la medición de AF y tiempo sedentario a través de cuestionarios autorreportados. Si bien GPAQ v2 ha sido validado internacionalmente ${ }^{19,20}$, existe evidencia que la medición de los niveles de sedentarismo a través de este cuestionario no es representativa en población chilena ${ }^{26}$. A su vez, la ENS 2009-2010 carece de un diseño de seguimiento longitudinal o de estudio de control con distribución aleatoria, por lo que no permite establecer una relación de causa-efecto. También cabe mencionar el efecto de "causalidad reversa", que plantea que los sujetos que reportaron mayor tiempo sentado o un mayor nivel de adiposidad 
fue debido a que ya presentaban algún tipo de patología, ya sea DMT2 u otra alteración cardiovascular, y por eso fueron clasificados dentro de estos grupos de riesgo, por ende, estudios de intervención o estudios longitudinales son necesarios para corroborar una asociación de causa-efecto de estos resultados.

Finalmente, es importante recalcar que todos los esfuerzos orientados en la promoción de un estilo de vida saludable y la prevención de enfermedades podrían ser más efectivos que el tratamiento posterior de las patologías y sus complicaciones. Iniciar con pequeñas acciones, tales como pequeñas pausas activas que impliquen movimientos en los puestos de trabajo, como lo han demostrado diversos autores ${ }^{27-29}$, podrían contribuir en la disminución del riesgo de DMT2 y otras enfermedades crónicas no transmisibles.

Agradecimientos: Se agradece de manera especial a todos los participantes de la ENS 20092010, al equipo profesional de la Escuela de Salud Pública, de la Facultad de Medicina de la Pontificia Universidad Católica de Chile, quienes desarrollaron y aplicaron la Encuesta Nacional de Salud y al Ministerio de Salud del Gobierno de Chile.

\section{Referencias}

1. Rawshani A, Rawshani A, Franzen S, Eliasson B, Svensson AM, Miftaraj M, et al. Mortality and Cardiovascular Disease in Type 1 and Type 2 Diabetes. NEJM 2017; 376 (15): 1407-18.

2. Association AD. Diagnosis and classification of diabetes mellitus. Diabetes Care 2014; 37 (Supplement 1): S81-S90.

3. Boles A, Kandimalla R, Reddy PH. Dynamics of diabetes and obesity: Epidemiological perspective. Biochim Biophys Acta 2017; 1863 (5): 1026-36.

4. WHO. Diabetes. Nota descriptiva. World Health Organization. 2017. Available: http://www.who.int/mediacentre/factsheets/fs312/es/.

5. da Rocha Fernandes J, Ogurtsova K, Linnenkamp U, Guariguata L, Seuring T, Zhang P, et al. IDF Diabetes Atlas estimates of 2014 global health expenditures on diabetes. Diabetes Res Clin Pract 2016; 117: 48-54.

6. Guariguata L, Whiting DR, Hambleton I, Beagley J, Linnenkamp U, Shaw JE. Global estimates of diabetes prevalence for 2013 and projections for 2035. Diabetes Res Clin Pract 2014; 103 (2): 137-49.

7. MINSAL. Encuesta Nacional de Salud 2009-2010.
Chile: Ministerio de Salud; 2010. Available: http:// www.minsal.cl/portal/url/item/bcb03d7bc28b64dfe040010165012d23.pdf.

8. MINSAL. Encuesta Nacional de Salud 2016-2017. Ministerio de Salud de Chile. 2017. Available: http:// www.minsal.cl/wp-content/uploads/2017/11/ENS-201617_PRIMEROS-RESULTADOS.pdf.

9. Cristi-Montero C, Celis-Morales C, Ramírez-Campillo R, Aguilar-Farías N, Álvarez C, Rodríguez-Rodríguez F. ¡Sedentarismo e inactividad física no son lo mismo!: una actualización de conceptos orientada a la prescripción del ejercicio físico para la salud. Rev Med Chile 2015; 143: 1089-90.

10. Henson J, Dunstan DW, Davies MJ, Yates T. Sedentary behaviour as a new behavioural target in the prevention and treatment of type 2 diabetes. Diabetes Metab Res Rev 2016; 32 Suppl 1: 213-20.

11. Matthews CE, Chen KY, Freedson PS, Buchowski MS, Beech BM, Pate RR, et al. Amount of time spent in sedentary behaviors in the United States, 2003-2004. Am J Epidemiol 2008; 167 (7): 875-81.

12. Joseph JJ, Echouffo-Tcheugui JB, Golden SH, Chen H, Jenny NS, Carnethon MR, et al. Physical activity, sedentary behaviors and the incidence of type 2 diabetes mellitus: the Multi-Ethnic Study of Atherosclerosis (MESA). BMJ Open Diabetes Res Care 2016; 4 (1): e000185.

13. Owen N, Healy GN, Matthews CE, Dunstan DW. Too much sitting: the population health science of sedentary behavior. Sport Sci R 2010; 38 (3): 105-13.

14. Rockette-Wagner B, Edelstein S, Venditti EM, Reddy D, Bray GA, Carrion-Petersen ML, et al. The impact of lifestyle intervention on sedentary time in individuals at high risk of diabetes. Diabetologia 2015; 58 (6): 1198202.

15. Celis-Morales C, Salas C, Alduhishy A, Sanzana R, Martínez MA, Leiva A, et al. Socio-demographic patterns of physical activity and sedentary behaviour in Chile: results from the National Health Survey 2009-2010. J Public Health-UK 2016; 38 (2): e98-e105.

16. Pugazhenthi S, Qin L, Reddy PH. Common neurodegenerative pathways in obesity, diabetes, and Alzheimer's disease. Biochim Biophys Acta 2017; 1863 (5): 1037-45.

17. WHO. Obesity: Preventing and managing the Global Epidemic. 2000. Available: http://www.who.int/nutrition/publications/obesity/WHO_TRS_894/en/

18. WHO. Global Physical Activity Questionnaire: GPAQ version 2.0. World Health Organization; 2009. Available: http://www.who.int/ncds/surveillance/steps/ GPAQ\%20Instrument $\% 20$ and $\% 20$ Analysis $\% 20$ Guide\%20v2.pdf. 
19. Bull FC, Maslin TS, Armstrong T. Global Physical Activity Questionnaire (GPAQ): Nine Country Reliability and Validity Study. J Phys Act Health 2009; 6 (6): 790804.

20. Hoos T, Espinoza N, Marshall S, Arredondo EM. Validity of the Global Physical Activity Questionnaire (GPAQ) in Adult Latinas. J Phys Act Health 2012; 9 (5): 698-705.

21. WHO. Global recommendations on physical activity for health. World Health Organization; 2010.

22. Morris JN, Heady JA, Raffle PA, Roberts CG, Parks JW. Coronary heart-disease and physical activity of work. Lancet (London, England) 1953; 265 (6796): 1111-20; concl.

23. Turi BC, Monteiro HL, Ribeiro Lemes I, Codogno JS, Lynch KR, Asahi Mesquita CA, et al. TV viewing time is associated with increased all-cause mortality in Brazilian adults independent of physical activity. Scand J Med Sci Spor 2017.

24. van der Berg JD, Stehouwer CD, Bosma H, van der Velde JH, Willems PJ, Savelberg HH, et al. Associations of total amount and patterns of sedentary behaviour with type 2 diabetes and the metabolic syndrome: The Maastricht Study. Diabetologia 2016; 59 (4): 709-18.

25. Zhang P, Zhang X, Brown J, Vistisen D, Sicree R, Shaw J, et al. Global healthcare expenditure on diabetes for 2010 and 2030. Diabetes Res Clin Pract 2010; 87 (3): 293-301.

26. Aguilar-Farias N, Leppe Zamora J. Is a single question of the Global Physical Activity Questionnaire (GPAQ) valid for measuring sedentary behaviour in the Chilean population? J Spor Sci 2016; 1-6.

27. Healy GN, Matthews CE, Dunstan DW, Winkler EA, Owen N. Sedentary time and cardio-metabolic biomarkers in US adults: NHANES 2003-06. Eur Heart J 2011; 32 (5): 590-7.

28. Peddie MC, Bone JL, Rehrer NJ, Skeaff CM, Gray AR, Perry TL. Breaking prolonged sitting reduces postprandial glycemia in healthy, normal-weight adults: a randomized crossover trial. Am J Clin Nutr 2013; 98 (2): 358-66.

29. Healy GN, Dunstan DW, Salmon J, Cerin E, Shaw JE, Zimmet PZ, et al. Breaks in sedentary time: beneficial associations with metabolic risk. Diabetes Care 2008; 31 (4): 661-6. 